\title{
Dipeptidyl Peptidase-4 Inhibition in Patients with Type 2 Diabetes Treated with Saxagliptin, Sitagliptin, or Vildagliptin
}

\author{
Daniel A. Tatosian • Ying Guo • Andrea K. Schaeffer • \\ Natalia Gaibu • Serghei Popa • Aubrey Stoch • \\ Ronald B. Langdon • Eunkyung A. Kauh
}

To view enhanced content go to www.diabetestherapy-open.com

Received: July 31, 2013 / Published online: October 26, 2013

(c) The Author(s) 2013. This article is published with open access at Springerlink.com

\section{ABSTRACT}

Introduction: Saxagliptin, sitagliptin, and vildagliptin are dipeptidyl peptidase-4 (DPP-4) inhibitors widely approved for use in patients with type 2 diabetes. Using a crossover design, the present study compared trough levels of DPP-4 inhibition provided by these agents in a single cohort of patients with type 2 diabetes.

Methods: This was a randomized, placebocontrolled, open-label, five-period crossover study. Eligible patients were 18-65 years of age, either treatment-naïve or off prior

Clinicaltrials.gov \#NCT01582308.

D. A. Tatosian - Y. Guo - A. K. Schaeffer - A. Stoch · R. B. Langdon · E. A. Kauh $(\bowtie)$

Merck \& Co., Inc., One Merck Drive, P.O. Box 100, Whitehouse Station, NJ 08889-0100, USA

e-mail: eunkyung_kauh@merck.com

N. Gaibu $\cdot$ S. Popa

ARENSIA Exploratory Medicine, Republican Clinical Hospital, N. Testemitanu str, nr 29, Chişinău, Republic of Moldova

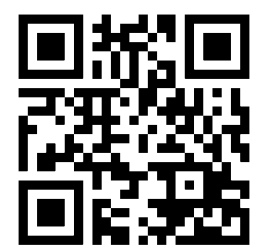

Enhanced content for this article is available on the journal web site: www.diabetestherapy-open.com antihyperglycemic agent therapy for at least 6 or 12 weeks (depending on the prior therapy), and had glycated hemoglobin $\left(\mathrm{HbA}_{1 \mathrm{C}}\right) \geq 6.5 \%$ and $\leq 10.0 \%$. In separate study periods, patients received $5 \mathrm{mg}$ saxagliptin q.d. (saxa-5), $100 \mathrm{mg}$ sitagliptin q.d. (sita-100), $50 \mathrm{mg}$ vildagliptin q.d. (vilda-50-q.d.), $50 \mathrm{mg}$ vildagliptin b.i.d. (vilda50-b.i.d.), or placebo for 5 days. The primary endpoint was trough \%DPP-4 inhibition, derived by comparing DPP- 4 activity $24 \mathrm{~h}$ after the Day-5 morning dose with predose activity in the same period and analyzed using a linear mixed-effects model with fixed-effects terms for treatment and period.

Results: Mean (range) baseline $\mathrm{HbA}_{1 \mathrm{C}}$ was $7.4 \%$ (6.4-9.0\%; $N=22$ ). Least-squares (LS) mean trough \%DPP-4 inhibition was 73.5\%, 91.7\%, $28.9 \%, 90.6 \%$, and $3.5 \%$ after saxa-5, sita-100, vilda-50-q.d., vilda-50-b.i.d., and placebo, respectively. In patients treated with sita-100, the LS-mean difference in trough \%DPP-4 inhibition was $18.2 \%$ greater than with saxa-5 $(p<0.001), 62.9 \%$ greater than with vilda-50q.d. $(p<0.001), 1.1 \%$ greater than with vilda50-b.i.d. $(p=0.128)$, and $87.8 \%$ greater than with placebo $(p<0.001)$. Mean \%DPP-4 inhibition was nearly maximal at $12 \mathrm{~h}$ 
postdose regardless of active treatment. Thus, these between-group comparisons at trough primarily reflected differences in duration of action. Adverse events reported during the study were transient and mild or moderate in intensity.

Conclusion: Once daily treatment with sitagliptin provided trough DPP-4 inhibition significantly greater than saxagliptin or vildagliptin administered once daily, and similar to that provided by vildagliptin administered twice daily.

Keywords: Dipeptidyl peptidase-4; Enzyme inhibition; Saxagliptin; Sitagliptin; Type 2 diabetes; Vildagliptin

\section{INTRODUCTION}

Dipeptidyl peptidase-4 (DPP-4) inhibitors improve glycemic control in patients with type 2 diabetes mellitus (T2DM) by increasing circulating levels of incretins, endogenous gutderived peptide hormones that enhance insulin secretion and suppress glucagon release in a glucose-dependent manner [1-4]. Several DPP-4 inhibitors are widely approved for use as oral antihyperglycemic agents in treating patients with T2DM. All have been shown to provide significant improvements in standard indices of glycemic control [5]. There may be important differences between them, however, in the extent to which they provide sustained pharmacodynamic (PD) action (DPP-4 inhibition) throughout their recommended dosing cycles. The existing PD data on these agents have come from separate studies in which each agent was evaluated individually, with different study populations and dosing regimens (single versus multiple dose administration) [6-11]. There is a need, therefore, for comparison of levels of DPP-4 inhibition between different agents within a single study in which conditions are standardized. There also is a need to minimize the impact of dilution and substrate competition artifacts that may be introduced in the process of assaying DPP-4 inhibition ex vivo [9, 11-14] and prevent meaningful comparison between agents even when tested in parallel within the same study.

Reagent solutions must be added to plasma in order to assay DPP-4 activity. As a result, plasma drug concentrations will be lower in an ex vivo assay than they are in vivo and this dilution effect may lead to underestimation of levels of DPP-4 inhibition. The impact of this effect will vary depending on the rate at which a DPP-4 inhibitor dissociates from its binding site on the enzyme, the amount of plasma dilution, and the length of time between the addition of reagents and the measurement of enzyme activity. In addition, there is the potential for ex vivo underestimation of DPP-4 inhibition due to substrate competition. If inhibition is assessed with substrate present at a level that saturates the enzyme, then competitive DPP-4 inhibitors are likely to appear to be less effective than they are under physiological conditions in vivo, where endogenous substrates are likely to be present at subsaturating concentrations.

Measurement of DPP-4 inhibition by sitagliptin is expected to be especially sensitive to these two artifacts because binding between sitagliptin and DPP-4 is competitive and rapidly reversible. When plasma containing sitagliptin is diluted, the half-life $\left(t_{1 / 2}\right)$ for readjustment to the new equilibrium for DPP- 4 binding is $<2 \mathrm{~min}$ [14], an interval that is brief compared with the length of time over which assays of plasma DPP-4 are typically run. Measurements of DPP-4 inhibition by vildagliptin may also be sensitive to the dilution artifact because the $t_{1 / 2}$ for 
dissociation of vildagliptin from DPP-4 is in the order of $3.5 \mathrm{~min}$ [14]. Measurements of DPP-4 inhibition by saxagliptin and an active metabolite, 5-hydroxy-saxagliptin, are likely to be relatively insensitive to this dilution effect because these molecular species dissociate slowly from DPP-4, with $t_{1 / 2}$ values of 50 and $23 \mathrm{~min}$, respectively [14]. Given these differences in binding kinetics, it follows that valid comparisons between these agents can only be made when DPP-4 inhibition is measured using an assay condition that minimizes the impact of plasma dilution. In the present study, we have directly compared profiles of DPP-4 inhibition over time in patients with T2DM treated with saxagliptin, sitagliptin, and vildagliptin using an assay in which plasma was diluted by only $11 \%$, a level that is likely to have only a minimal effect on outcome, regardless of binding kinetics. Using a randomized, placebo-controlled, openlabel, crossover design, patients in a single cohort were treated in successive study periods for 5 days (so that all agents could reach steadystate conditions) with the highest recommended doses for each agent. Pharmacokinetic (PK) and DPP-4 inhibition data were obtained at various times throughout these treatments and for an additional 4 days after the last doses were given. The prespecified primary endpoint was the 'trough' level of DPP-4 inhibition measured after patients received their final doses in each study period. It was assessed at $24 \mathrm{~h}$ postdose in subjects being treated with study drugs q.d. and at $12 \mathrm{~h}$ postdose in one study period in which patients were treated b.i.d. (with vildagliptin).

\section{METHODS}

This study was performed in accordance with all local laws and regulations, the Declaration of Helsinki of 1975 , as revised in 2000, and guidelines established by the International Conference on Harmonization Good Clinical Practice. The protocol was approved by the National Ethics Committee Clinical Research of Medicines and New Methods of Treatment (Chisinau, Moldova). Prior to participating, all patients provided written informed consent. The study was performed at the Clinical Republican Hospital (Chisinau, Moldova) between June and December, 2012.

\section{Patients}

Eligible patients were male or female, 18-65 years of age, and had T2DM with $\mathrm{HbA}_{1 \mathrm{C}}$ values $\geq 6.5 \%$ and $\leq 10.0 \%$ either while treatment-naïve or following washout of prior oral antihyperglycemic medications for $\geq 6$ weeks ( 12 weeks for patients previously treated with peroxisome proliferator-activated receptor- $\gamma$ agonists, insulin, DPP-4 inhibitors, or glucagon-like peptide-1 analogs). Patients with fasting plasma glucose $>200 \mathrm{mg} / \mathrm{dL}(11.1 \mathrm{mmol} / \mathrm{L})$ or estimated creatinine clearance $\leq 60 \mathrm{~mL} / \mathrm{min}$ were excluded. Concomitant, stable use of medications for pre-existing hypertension and dyslipidemia was permitted. Use of medications, nutritional supplements, and dietary products known to be cytochrome P3A4/5 (CYP3A4/5) inhibitors, CYP3A4/5 inducers, P-glycoprotein-1 inhibitors, or human organic anion transporter 3 inhibitors was prohibited.

\section{Study Design}

This was an open-label, placebo-controlled, fiveperiod crossover study in which each patient received in separate periods $5 \mathrm{mg}$ saxagliptin q.d., $100 \mathrm{mg}$ sitagliptin q.d., $50 \mathrm{mg}$ vildagliptin q.d., $50 \mathrm{mg}$ vildagliptin b.i.d. (50 mg every $12 \mathrm{~h}$ ), and placebo q.d. These specific doses were tested because each is a highest 
recommended dose. With vildagliptin, $50 \mathrm{mg}$ b.i.d. is a highest dose approved for use as monotherapy and $50 \mathrm{mg}$ q.d. is the recommended dose approved for use in combination with a sulfonylurea. Assignment to treatment sequences was randomized using a computer-generated schedule. Treatments were administered for 5 days and separated by $\geq 10$ days.

\section{Procedures}

Patients received nutritional and exercise counseling and were provided with glucose meters for home blood glucose monitoring. They were instructed to perform fingerstick glucose measurements $5 \mathrm{~min}$ before and $2 \mathrm{~h}$ after each meal, and to contact the study-site staff in the event that any blood glucose value was $<70 \mathrm{mg} / \mathrm{dL}(3.9 \mathrm{mmol} / \mathrm{L})$ or any fasting blood glucose value was $>200 \mathrm{mg} / \mathrm{dL}$ $(11.1 \mathrm{mmol} / \mathrm{L})$. It was prespecified that patients would be discontinued from the study in the event of having more than one occurrence of fasting blood glucose $>200 \mathrm{mg} /$ $\mathrm{dL}$ or a single occurrence of any blood glucose measurement $>250 \mathrm{mg} / \mathrm{dL}(14 \mathrm{mmol} / \mathrm{L})$.

Patients were domiciled in the clinic in each study period from Day -1 (the day before the first dose) through the morning of Day 1. They were also domiciled from the evening of Day 4 through the morning of Day 6 (and provided with standard meals and snacks during this time). Approximately 14 days after receiving final doses of study drug, patients returned for a follow-up visit. All doses were administered in the clinic, either during outpatient visits or while patients were domiciled. The first dose was preceded by an overnight fast of at least $8 \mathrm{~h}$ and study medications were administered with approximately $240 \mathrm{~mL}$ of water. Evening doses of vildagliptin were given without regard to timing of consumption of food or drink.

Blood samples for PD analysis were collected into Vacutainer ${ }^{\mathrm{TM}}$ EDTA tubes (Becton Dickinson, Franklin Lakes, NJ, USA) predose on Days 1-5 and 12, 24, 36, 48, $96 \mathrm{~h}$ after the final morning dose on Day 5. Plasma was then isolated by centrifugation, divided into aliquots, and stored at $-80{ }^{\circ} \mathrm{C}$ until analysis. Blood samples for PK analysis were collected predose on Days 1-5 and 0.5, 1, 2, 4, 8, 12, 16, $24,36,48$, and $96 \mathrm{~h}$ following the final morning dose on Day 5. During treatment with vildagliptin b.i.d., additional samples were collected 13 and $14 \mathrm{~h}$ after the final morning dose on Day 5. For analysis of saxagliptin, 5-hydroxy-saxagliptin, and sitagliptin concentrations, samples were collected into Vacutainer $^{\mathrm{TM}}$ K2EDTA tubes (Becton Dickinson) and plasma was stored at $-20^{\circ} \mathrm{C}$. For analysis of vildagliptin, samples were collected into Vacutainer ${ }^{\mathrm{TM}}$ P800 tubes (Becton Dickinson) and plasma was stored at $-80^{\circ} \mathrm{C}$.

\section{Measurement of Plasma DPP-4 Activity}

Assay of plasma DPP-4 activity was performed at PPD Bioanalytical Lab (Richmond, VA, USA). In this assay, $90 \mu \mathrm{L}$ of plasma was combined with $10 \mu \mathrm{L} \quad$ of $\quad 100 \mu \mathrm{M} \quad$ glycyl-prolyl-aminomethylcoumarin hydrobromide (Bachem, Torrance, CA, USA). The final concentration of substrate was therefore $10 \mu \mathrm{M}$, a level much lower than the $K_{\mathrm{M}}$ of human DPP-4 for this substrate (approximately $50 \mu \mathrm{M}$ ). Inhibition of DPP-4 was thus assayed under conditions designed to minimize the effects of plasma dilution and substrate competition.

The assay solutions were incubated at $37^{\circ} \mathrm{C}$ in a black Immuno Plate (Part \# 7205, Thermo 
Scientific, Waltham, MA, USA) on a SpectraMax Gemini EM $^{\mathrm{TM}}$ Fluorescence Microplate Reader (Molecular Devices, Sunnyvale, CA, USA). Data were analyzed using SoftmaxPro ${ }^{\circledR}$ applications (Molecular Devices, Sunnyvale, CA, USA). Formation of reaction product was monitored by measuring the increase in relative fluorescence at $460 \mathrm{~nm}$ at 45 -s intervals (using $360 \mathrm{~nm}$ as the excitation wavelength). Enzyme activity $(A)$ was defined as the slope of this increase [in relative fluorescent units per seconds (RFU/s)] measured over the first $5 \mathrm{~min}$ of incubation. In a preliminary analysis, it was determined that $1 \mathrm{RFU} / \mathrm{s}$ was equivalent to product formation at a rate of approximately $0.2 \mathrm{pmol} / \mathrm{s}$.

Percent inhibition of DPP-4 was calculated as $100 \times\left(1-A_{t} / A_{0}\right)$, where $A_{0}$ was the enzyme activity measured predose and $A_{t}$ was the activity measured postdose at time $t$ in the same treatment period. The lower limit of quantification (LOQ) for measurement of $A$ was defined as the lowest level at which the imprecision (coefficient of variation) of measurements remained $<20 \%$. The LOQ was regarded as constant across all subjects and study periods. When $A_{t}$ did not exceed the LOQ, it was assigned the value of the LOQ (0.366 $\mathrm{RFU} / \mathrm{s})$.

\section{Pharmacokinetic Analyses}

Plasma concentrations of saxagliptin, 5-hydroxy-saxagliptin, sitagliptin, and vildagliptin were analyzed by liquid chromatography-tandem mass spectrometry at Pharmanet Canada, Inc. (now Inventive Health Clinical, Québec City, Québec, Canada). The analysis of sitagliptin was performed as previously described [15]. For saxagliptin and 5-hydroxy-saxagliptin, the validated calibration ranges were $100-50,000 \mathrm{pg} / \mathrm{mL}$ and
200-100,000 pg/mL, respectively. For sitagliptin, the validated range was $1.00-1,000 \mathrm{ng} / \mathrm{mL}$. For vildagliptin, it was $1.00-2,000 \mathrm{ng} / \mathrm{mL}$.

Non-compartmental PK analysis was performed with actual sampling times using the WinNonlin ${ }^{\mathrm{TM}}$ program (Professional, version 5.0.1; Pharsight ${ }^{\circledR}$, Mountain View, CA, USA). Areas under the curve (AUCs) for concentration versus time were calculated using the linear trapezoidal method for ascending concentrations and the log trapezoidal method for descending concentrations. The terminal elimination rate constant $(\lambda z)$ was calculated as the negative of the slope of the terminal phase of the log-linear concentration versus time curve. These analyses included at least three terminal points. Apparent terminal $t_{1 / 2}$ was calculated as $\ln (2) / \lambda z$

\section{Statistical Methods}

All available data were included in the analysis (including data from patients who did not complete the study but had at least one measurement). Levels of DPP-4 inhibition were analyzed using a linear mixed-effects model with fixed-effects terms for treatment and period. Data were transformed to a log-percent scale for analysis and then back-transformed to a linear scale for reporting. Baseline was defined in each period as the predose value on Day 1 . Inference testing was one-sided and significance was defined as $p<0.05$.

A heterogeneous compound symmetry covariance matrix was used to allow for unequal treatment variances and to model correlation between different treatment effects within the same subject via the REPEATED Statement $^{\circledR}$ in SAS PROC MIXED ${ }^{\circledR}$ (SAS Institute, Inc., Cary, NC, USA). Kenward and 
Roger's method [16] was used to calculate the denominator degrees of freedom for the fixed effects. It was assumed that time and carryover effects were negligible. One-sided 95\% confidence intervals (CIs) for betweentreatment differences in trough DPP-4 inhibition were calculated using the linear mixed-effects model mentioned above.

The primary hypothesis was that treatment of patients with T2DM with $100 \mathrm{mg}$ sitagliptin q.d. provides significantly greater trough DPP-4 inhibition than treatment with $5 \mathrm{mg}$ saxagliptin or $50 \mathrm{mg}$ vildagliptin q.d. It was prespecified that if the lower bound of the onesided 95\% CI for the difference in trough DPP-4 inhibition between sitagliptin treatment and a comparator was $>0$, then this hypothesis would be confirmed. In addition, 95\% CIs were calculated for the differences between all other pairs of treatments in this study and summary statistics were calculated for each treatment and timepoint in the study.

Based on historical observations of variability, it was calculated that a sample comprising 15 completing patients would provide at least $83 \%$ power $(\alpha=0.05$, onesided) to detect a $20 \%$ difference (absolute percentage points) between treatments in trough DPP-4 inhibition.

\section{RESULTS}

The randomized cohort included 12 women and 10 men (all Caucasian) with a mean (range) age of 55 (43-64) years and body mass index of $32(26-39) \mathrm{kg} / \mathrm{m}^{2}$. Mean (range) baseline $\mathrm{HbA}_{1 \mathrm{C}}$ was $7.4 \%(6.4-9.0 \%)$.

Five patients discontinued prior to study completion. One withdrew consent, one was determined after randomization not to have met eligibility criteria, and three patients had elevated blood glucose concentrations that met the prespecified criteria for discontinuation. These incidents of elevated blood glucose occurred primarily prior to dosing with active treatments (i.e., during washout periods). The investigator did not consider these to be adverse events (AEs).

Predose levels of DPP-4 activity $\left(A_{0}\right)$ were 3.70-6.36 RFU/s (10.1- to 17.4-fold greater than the LOQ). Thus, the upper limits for quantitation of DPP-4 inhibition in individual measurements were between $90 \%$ and $94 \%$. Actual values varied for each subject and treatment period based on the baseline DPP-4 activity measured in that period.

\section{Plasma DPP-4 Inhibition}

Trough levels of DPP-4 inhibition were generally stable by the morning of Day 3 during all active treatments (Fig. 1a) and each of these provided nearly maximal inhibition of DPP-4 at $12 \mathrm{~h}$ postdose as assessed on Day 5 (Fig. 1b). Two of the treatments, sitagliptin and vildagliptin b.i.d., provided nearly maximal inhibition at $24 \mathrm{~h}$ postdose as determined predose on Days 1-5 and also on the morning of Day 6, when the primary endpoint was assessed. With these treatments, LS-mean trough DPP-4 inhibition on Day 6 was $91.7 \%$ and 90.6\%, respectively (Table 1 ). The difference between them was not significant $(p=0.128)$.

Treatment with either saxagliptin or vildagliptin q.d. provided significantly $(p<0.001)$ less trough DPP-4 inhibition than treatment with either sitagliptin or vildagliptin b.i.d. With saxagliptin, the LS-mean trough DPP-4 inhibition on Day 6 was $73.5 \%$ (18.2\% and $17.1 \%$ less than with sitagliptin and vildagliptin b.i.d., respectively). With vildagliptin q.d., it was $28.9 \%(62.9 \%$ and $61.8 \%$ less than with sitagliptin and 

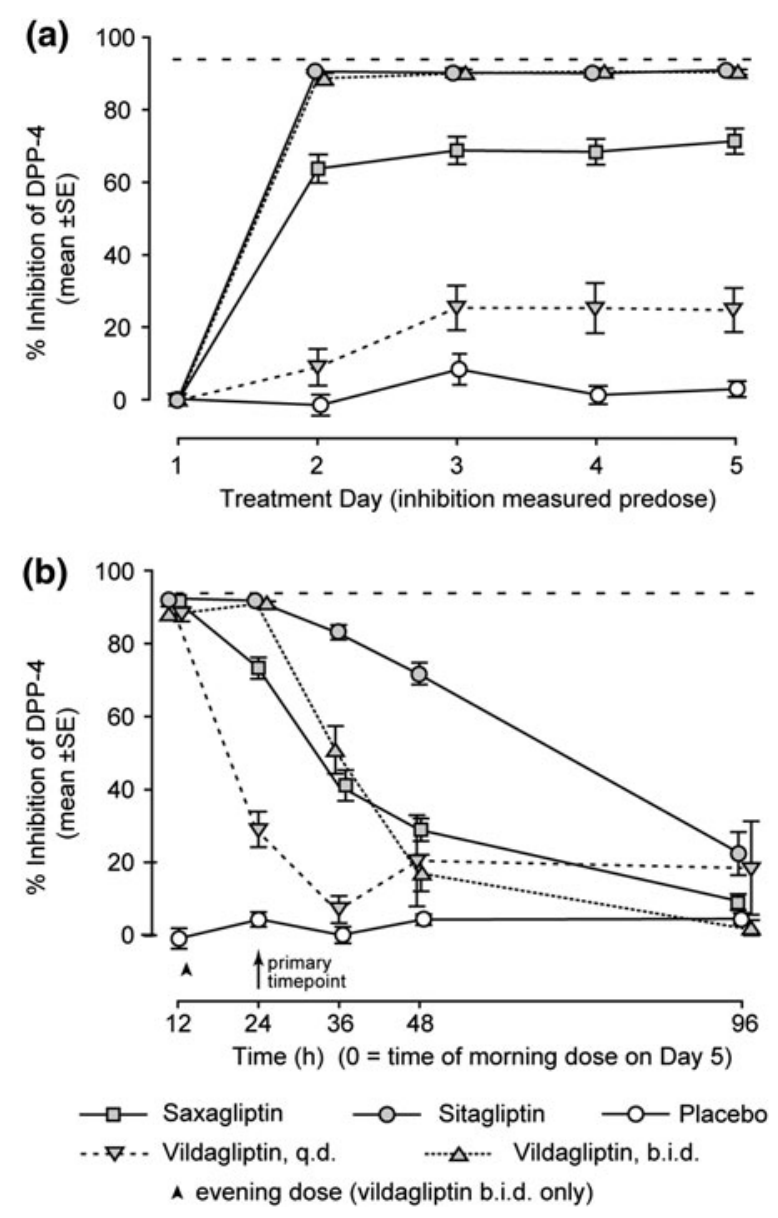

Fig. 1 Mean ( \pm standard error) percent dipeptidyl peptidase-4 (DPP-4) inhibition over time in plasma collected a predose on Days $1-5$ of treatment with $5 \mathrm{mg}$ saxagliptin q.d., $100 \mathrm{mg}$ sitagliptin q.d., $50 \mathrm{mg}$ vildagliptin q.d., $50 \mathrm{mg}$ vildagliptin b.i.d., and placebo, and $\mathbf{b}$ at the indicated times on Days 5-9. The measurement at $12 \mathrm{~h}$ postdose on Day 5 was obtained before the second vildagliptin b.i.d. dose was administered. The dashed horizontal lines indicate 94\% inhibition, the maximum level of DPP-4 inhibition that was quantifiable under the assay conditions used in this study. $S E$ standard error

vildagliptin b.i.d., respectively). Treatment with saxagliptin resulted in $44.6 \%$ greater trough DPP-4 inhibition on Day 6 than treatment with vildagliptin q.d. $(p<0.001)$.

At 36 and $48 \mathrm{~h}$ after morning doses were given on Day 5, the mean levels of DPP-4 inhibition following sitagliptin treatment were $83 \%$ and $72 \%$, respectively (Fig. 1b). Following vildagliptin b.i.d., saxagliptin, and vildagliptin q.d., the mean levels of DPP-4 inhibition at these times were $50 \%$ and $17 \%, 41 \%$ and $29 \%$, and $7 \%$ and $21 \%$, respectively. At the final timepoint ( $96 \mathrm{~h}$ after the morning dose on Day $5)$, mean DPP-4 inhibition was $<25 \%$ with all treatments.

\section{Pharmacokinetic Observations}

Observed values for $\mathrm{AUC}_{0-24 \mathrm{~h}}$, maximal concentration $\left(C_{\max }\right)$, concentration at $24 \mathrm{~h}$ $\left(C_{24} \mathrm{~h}\right)$, and other PK parameters are shown in Table 2. All dosing regimens appeared to result in steady-state plasma concentrations of parent drugs by Day 3 (data not shown). Plasma concentrations of 5-hydroxy-saxagliptin appeared to reach steady state by Day 5 .

\section{Safety}

The study treatments were generally well tolerated. In total, 10 AEs were reported in 7 of the 22 patients enrolled. Headache was the most commonly reported $\mathrm{AE}$ (six events reported in three patients). Five AEs were considered to be study related. These included four events of headache (two that were attributed to saxagliptin treatment and two that were attributed to sitagliptin treatment) and one event of influenza that was attributed to saxagliptin treatment. All AEs were reported to be mild $(n=6)$ or moderate $(n=4)$ in intensity and transient in nature. There were no deaths, no serious AEs, and no AEs that led to withdrawal from the study.

\section{Other Observations}

In any period in which a patient was administered placebo, plasma concentrations of saxagliptin, 5-hydroxy-saxagliptin, sitagliptin, 
Table 1 Percent inhibition of dipeptidyl peptidase- 4 activity $24 \mathrm{~h}$ after final morning doses in patients with type 2 diabetes mellitus treated for 5 days with $5 \mathrm{mg}$ saxagliptin q.d., $100 \mathrm{mg}$ sitagliptin q.d., $50 \mathrm{mg}$ vildagliptin q.d., $50 \mathrm{mg}$ vildagliptin b.i.d., or placebo

\begin{tabular}{lcr}
\hline Treatment & $\boldsymbol{N}$ & $\begin{array}{c}\text { Least-squares } \\
\text { mean }(\mathbf{9 5 \%} \mathbf{C I})^{\mathbf{a}}\end{array}$ \\
\hline Saxagliptin q.d. & 20 & $73.5(66.6,79.0)$ \\
Sitagliptin q.d. & 17 & $91.7(91.4,92.1)$ \\
Vildagliptin q.d. & 18 & $28.9(17.9,38.4)$ \\
Vildagliptin b.i.d. & 17 & $90.6(88.9,92.1)$ \\
Placebo & 17 & $3.5(-0.7,7.5)$ \\
\hline Comparison & Difference (90\% CI) & p value \\
\hline Sitagliptin q.d. vs. saxagliptin q.d. & $18.2(15.0,21.7)$ & $<0.001$ \\
Sitagliptin q.d. vs. vildagliptin q.d. & $62.9(58.2,67.7)$ & $<0.001$ \\
Sitagliptin q.d. vs. vildagliptin b.i.d. & $1.1(-0.1,2.3)$ & 0.128 \\
Sitagliptin q.d. vs. placebo & $88.2(85.8,90.8)$ & $<0.001$ \\
Saxagliptin q.d. vs. vildagliptin q.d. & $44.6(34.5,55.2)$ & $<0.001$ \\
Saxagliptin q.d. vs. vildagliptin b.i.d. & $-17.1(-21.2,-13.3)$ & $<0.001$ \\
Saxagliptin q.d. vs. placebo & $70.0(58.5,82.2)$ & $<0.001$ \\
Vildagliptin q.d. vs. vildagliptin b.i.d. & $-61.8(-68.8,-55.2)$ & $<0.001$ \\
Vildagliptin q.d. vs. placebo & $25.4(15.4,35.5)$ & $<0.001$ \\
Vildagliptin b.i.d. vs. placebo & $87.1(80.1,94.6)$ & $<0.001$ \\
\hline$C I$ confidence interval & & \\
a Back-transformed from a log scale & & \\
b Between-treatment differences in percent inhibition; positive values favor the first treatment listed at left &
\end{tabular}

and vildagliptin were measured predose on Day 1 and these were observed to be below the LOQ in all cases. These findings suggest that there was no carryover of drug concentration from one period to the next. Similarly, predose levels of DPP-4 inhibition on Day 1 were similar among patients in all treatment sequences suggesting no PD carryover, a conclusion that was further supported by the results of a sensitivity analysis in which predose measurements of DPP-4 activity in Period 1, Day 1 were used as the baseline for all subsequent periods (data not shown).

\section{DISCUSSION}

The findings from this study show that saxagliptin, sitagliptin, and vildagliptin differ significantly in the trough levels of DPP-4 inhibition that they provide when administered to patients with T2DM following recommended q.d. and b.i.d. dosing regimens. Sitagliptin and twice-daily vildagliptin each provided trough inhibition levels of approximately $90 \%$, the maximum inhibition level that was observed at any time in this study. In contrast, saxagliptin and once-daily 
Table 2 Plasma pharmacokinetic parameters for saxagliptin, 5-hydroxy-saxagliptin (5-OH-saxagliptin), sitagliptin, and vildagliptin following treatment for 5 days with saxagliptin $5 \mathrm{mg}$ q.d., sitagliptin $100 \mathrm{mg}$ q.d., vildagliptin $50 \mathrm{mg}$ q.d., and $50 \mathrm{mg}$ vildagliptin b.i.d. in patients with type 2 diabetes mellitus

\begin{tabular}{lllllllr}
\hline & $N$ & $\begin{array}{l}C_{\max } \\
(\mathbf{n M})^{\mathbf{a}}\end{array}$ & $\begin{array}{l}\boldsymbol{T}_{\max } \\
(\mathbf{h})^{\mathbf{b}}\end{array}$ & $\begin{array}{l}\boldsymbol{C}_{\mathbf{2 4 h}} \\
(\mathbf{n M})\end{array}$ & $\begin{array}{l}\text { Apparent } \boldsymbol{t}_{\mathbf{1} / \mathbf{2}} \\
(\mathbf{h})\end{array}$ & $\begin{array}{l}\mathbf{A U C}_{\mathbf{0 - 1 2 h}} \\
(\mathbf{n M ~ h})\end{array}$ & $\begin{array}{l}\mathbf{A U C}_{\mathbf{0 - 2 4 h}} \\
(\mathbf{n M ~ h})\end{array}$ \\
\hline Saxagliptin & 20 & $88.8(25.2)$ & $1(0.5-2)$ & $1.1(41.8)$ & $9.49(23.9)$ & $341(24.4)$ & $370(25.0)$ \\
5-OH-Saxagliptin & 20 & $113(35.1)$ & $2(1-4)$ & $4.7(27.8)$ & $14.7(10.9)$ & $674(31.7)$ & $784(31.7)$ \\
Sitagliptin & 20 & $724(31.3)$ & $4(0.5-8)$ & $84.4(36.3)$ & $12.0(31.4)$ & $5,340(24.9)$ & $7,070(25.1)$ \\
Vildagliptin q.d. & 18 & $586(37.0)$ & $1(0.5-8)$ & $<\mathrm{LOQ}^{\mathrm{c}}$ & $2.42(58.5)$ & $2,990(27.2)$ & $3,100(27.0)$ \\
Vildagliptin b.i.d. & 18 & $759(34.7)$ & $1(0.5-4)$ & $7.8(105.1)^{\mathrm{d}}$ & $3.41(42.2)$ & $3,720(28.9)^{\mathrm{e}}$ & $6,600(26.3)$ \\
\hline
\end{tabular}

a Except where indicated, the data are geometric means (\% coefficient of variation)

b Median (range)

c $>50 \%$ of samples were below the limit of quantitation

d Some samples were below the limit of quantitation; the arithmetic mean (\% coefficient of variation) is reported

e $N=17$

vildagliptin provided trough inhibition levels of approximately $75 \%$ and $30 \%$, respectively. Thus, it was observed that sitagliptin and twice-daily vildagliptin provided sustained, high levels of DPP-4 inhibition throughout $24 \mathrm{~h}$, whereas saxagliptin and once-daily vildagliptin did not.

The trough level of DPP-4 inhibition observed in this study following saxagliptin treatment corresponded well with the trough level of inhibition ( $\sim 70 \%)$ observed previously in a 2-week study in which patients were treated with saxagliptin $5 \mathrm{mg}$ q.d. [17]. To our knowledge, there are no published DPP-4 inhibition data from patients treated with multiple doses of $50 \mathrm{mg}$ vildagliptin b.i.d.; direct comparison between present and historical data is thus not possible. In a study in which single doses of 50 and $100 \mathrm{mg}$ vildagliptin were given to patients with T2DM, the levels of DPP-4 inhibition observed at $24 \mathrm{~h}$ postdose were approximately $14 \%$ and $35 \%$, respectively [7]. In the present study, the mean ( \pm standard error) level of DPP-4 inhibition $24 \mathrm{~h}$ after the first dose of vildagliptin $50 \mathrm{mg}$ q.d. was
$9.1 \pm 5.0 \%$ (Fig. 1). This value is reasonably consistent with the prior result.

In the present study, sitagliptin provided a greater trough level of DPP-4 inhibition than was previously observed in a Phase I trial in which patients with T2DM were administered a single dose of $200 \mathrm{mg}$ sitagliptin. In that study, plasma samples were diluted 2.5-fold prior to ex vivo assay and the level of DPP-4 inhibition measured at $24 \mathrm{~h}$ postdose was $80.1 \%$ [6]. The greater level of DPP-4 inhibition observed in the present study could be accounted for by the difference in plasma dilution. A statistical method has been described to correct for this dilution, and based on this method one would expect to observe $80 \%$ DPP-4 inhibition by sitagliptin after 2.5-fold dilution of plasma when the true level of inhibition in vivo is $\sim 96 \%$ [9]. We have not applied this correction in the present analysis because the extent of plasma dilution in our assay was sufficiently small $(11 \%)$ that its effect could reasonably be regarded as negligible.

The PK findings in the present study were generally consistent with those reported 
previously $[6,8,10]$. It was confirmed that all plasma drug concentrations were at steady state when the primary endpoint was measured and that PD effects were stable from Day 3 onwards. Hence, differences in drug exposure did not appear to play a role in the observed differences in PD effect.

One limitation of this study was that antihyperglycemic efficacy was not evaluated. Hence, uncertainty remains as to whether the observed differences in DPP-4 inhibition have meaningful implications for glycemic efficacy. However, a recent, model-based meta-analysis of published data found evidence for greater reductions in $\mathrm{HbA}_{1 \mathrm{C}}$ with more sustained DPP4 inhibition [18]. Increasing reductions in $\mathrm{HbA}_{1 \mathrm{C}}$ were found in this meta-analysis even as the weighted average DPP-4 inhibition increased beyond 90\%, supporting the concept that maintaining nearly complete inhibition of DPP-4 over entire dosing cycles may be important in order to maximize antihyperglycemic efficacy with this class of drugs. In addition, we note that the finding of similar DPP-4 inhibition during treatment in our comparison of once-daily sitagliptin with twicedaily vildagliptin is consistent with prior reports suggesting generally similar glycemic efficacy when these regimens are compared [19].

In a study comparing the efficacy of sitagliptin $100 \mathrm{mg}$ q.d. with saxagliptin $5 \mathrm{mg}$ q.d. as an addon to metformin for 18 weeks, the adjusted mean change from baseline in $\mathrm{HbA}_{1 \mathrm{C}}$ was $-0.62 \%$ versus $-0.52 \%$ for sitagliptin and saxagliptin, respectively [20]. Although this difference was within the study's prespecified limit for noninferiority for $\mathrm{HbA}_{1 \mathrm{C}}$, the sitagliptin group had a numerically greater adjusted mean change from baseline in fasting plasma glucose than the saxagliptin group $[-16.2 \mathrm{mg} / \mathrm{dL}(-0.90 \mathrm{mmol} / \mathrm{L})$ versus $\quad-10.8 \mathrm{mg} / \mathrm{dL} \quad(-0.60 \mathrm{mmol} / \mathrm{L})$, respectively]. The results of the present study suggest a possible mechanism for this glycemic outcome, in that sitagliptin $100 \mathrm{mg}$ provided significantly greater DPP-4 inhibition at $24 \mathrm{~h}$ postdose than saxagliptin $5 \mathrm{mg}$.

\section{CONCLUSION}

This study found that three approved DPP-4 inhibitors provided significantly different trough levels of DPP-4 inhibition when administered to patients with T2DM following recommended q.d. and b.i.d. dosing regimens. Maximal inhibition was sustained throughout $24 \mathrm{~h}$ in patients treated with sitagliptin $100 \mathrm{mg}$ once daily and vildagliptin $50 \mathrm{mg}$ twice daily. Trough DPP-4 inhibition was submaximal in patients treated with saxagliptin $5 \mathrm{mg}$ and vildagliptin $50 \mathrm{mg}$ once daily.

\section{ACKNOWLEDGMENTS}

The authors gratefully acknowledge the helpful comments and discussion provided by Manu Chakravarthy, Chan R. Beals, Samuel S. Engel, Patrick J. Larson, Omar F. Laterza, and Terri M. Kelly (all of Merck \& Co., Inc.) while this manuscript was in preparation. Eunkyung Kauh is the guarantor for this article, and takes full responsibility for the integrity of the work as a whole. Article processing charges for this study were funded by Merck \& Co., Inc.

Conflict of interest. Daniel A. Tatosian is an employee of Merck Sharp \& Dohme Corp., a subsidiary of Merck \& Co. Inc., the sponsor of this study, and may own stock and/or stock options. Ying Guo is an employee of Merck Sharp \& Dohme Corp. and may own stock and/ or stock options. Andrea K. Schaeffer is an employee of Merck Sharp \& Dohme Corp. and may own stock and/or stock options. Aubrey 
Stoch is an employee of Merck Sharp \& Dohme Corp. and may own stock and/or stock options. Ronald B. Langdon is an employee of Merck Sharp \& Dohme Corp. and may own stock and/ or stock options. Eunkyung A. Kauh is an employee of Merck Sharp \& Dohme Corp. and may own stock and/or stock options. Natalia Gaibu is an employee of Arensia Exploratory Medicine, which was contracted by Merck \& Co., Inc. to conduct this study. Serghei Popa is an employee of Arensia Exploratory Medicine.

Compliance with ethics guidelines. This study was performed in accordance with the Declaration of Helsinki of 1975, as revised in 2000, and guidelines established by the International Conference on Harmonization Good Clinical Practice. The protocol for this study was approved by the National Ethics Committee Clinical Research of Medicines and New Methods of Treatment (Chisinau, Moldova). Prior to participating, all patients provided written informed consent.

Open Access. This article is distributed under the terms of the Creative Commons Attribution Noncommercial License which permits any noncommercial use, distribution, and reproduction in any medium, provided the original author(s) and the source are credited.

\section{REFERENCES}

1. Holst JJ, Deacon CF. Inhibition of the activity of dipeptidyl-peptidase IV as a treatment for type 2 diabetes. Diabetes. 1998;47:1663-70.

2. Drucker DJ. Development of glucagon-like peptide1-based pharmaceuticals as therapeutic agents for the treatment of diabetes. Curr Pharm Des. 2001;7:1399-412.

3. Conarello SL, Li Z, Ronan J, et al. Mice lacking dipeptidyl peptidase IV are protected against obesity and insulin resistance. Proc Natl Acad Sci USA. 2003;100:6825-30.

4. Drucker DJ, Sherman SI, Gorelick FS, Bergenstal RM, Sherwin RS, Buse JB. Incretin-based therapies for the treatment of type 2 diabetes: evaluation of the risks and benefits. Diabetes Care. 2010;33:428-33.

5. Deacon CF. Dipeptidyl peptidase- 4 inhibitors in the treatment of type 2 diabetes: a comparative review. Diabetes Obes Metab. 2011;13:7-18.

6. Herman GA, Bergman A, Stevens C, et al. Effect of single oral doses of sitagliptin, a dipeptidyl peptidase-4 inhibitor, on incretin and plasma glucose levels after an oral glucose tolerance test in patients with type 2 diabetes. J Clin Endocrinol Metab. 2006;91:4612-9.

7. He YL, Wang Y, Bullock JM, et al. Pharmacodynamics of vildagliptin in patients with type 2 diabetes during OGTT. J Clin Pharmacol. 2007;47:633-41.

8. He YL, Sabo R, Campestrini J, et al. The effect of age, gender, and body mass index on the pharmacokinetics and pharmacodynamics of vildagliptin in healthy volunteers. $\mathrm{Br} \mathrm{J}$ Clin Pharmacol. 2008;65:338-46.

9. Alba M, Sheng D, Guan Y, et al. Sitagliptin $100 \mathrm{mg}$ daily effect on DPP-4 inhibition and compoundspecific glycemic improvement. Curr Med Res Opin. 2009;25:2507-14.

10. Boulton DW, Smith CH, Li L, Huang J, Tang A, LaCreta FP. Bioequivalence of saxagliptin/ metformin extended-release (XR) fixed-dose combination tablets and single-component saxagliptin and metformin XR tablets in healthy adult subjects. Clin Drug Investig. 2011;31:619-30.

11. Pospisilik JA, Stafford SG, Demuth HU, et al. Longterm treatment with the dipeptidyl peptidase IV inhibitor P32/98 causes sustained improvements in glucose tolerance, insulin sensitivity, hyperinsulinemia, and beta-cell glucose responsiveness in VDF (fa/fa) Zucker rats. Diabetes. 2002;51:943-50.

12. Edmondson SD, Mastracchio A, Duffy JL, et al. Discovery of potent and selective orally bioavailable beta-substituted phenylalanine derived dipeptidyl peptidase IV inhibitors. Bioorg Med Chem Lett. 2005;15:3048-52.

13. Matheeussen V, Lambeir AM, Jungraithmayr W, et al. Method comparison of dipeptidyl peptidase IV activity assays and their application in biological samples containing reversible inhibitors. Clin Chim Acta. 2012;413:456-62. 
14. Wang A, Dorso C, Kopcho L, et al. Potency, selectivity and prolonged binding of saxagliptin to DPP-4: maintenance of DPP4 inhibition by saxagliptin in vitro and ex vivo when compared to a rapidly-dissociating DPP-4 inhibitor. BMC Pharmacol. 2012;12:2.

15. Zeng $\mathrm{W}, \mathrm{Xu}$ Y, Constanzer $\mathrm{M}$, Woolf EJ. Determination of sitagliptin in human plasma using protein precipitation and tandem mass spectrometry. J Chromatogr B Anal Technol Biomed Life Sci. 2010;878:1817-23.

16. Kenward MG, Roger JH. Small sample inference for fixed effects from restricted maximum likelihood. Biometrics. 1997;53:983-97.

17. Tahrani AA, Piya MK, Barnett AH. Saxagliptin: a new DPP-4 inhibitor for the treatment of type 2 diabetes mellitus. Adv Ther. 2009;26:249-62.
18. Gibbs JP, Fredrickson J, Barbee T, et al. Quantitative model of the relationship between dipeptidyl peptidase-4 (DPP-4) inhibition and response: meta-analysis of alogliptin, saxagliptin, sitagliptin, and vildagliptin efficacy results. J Clin Pharmacol. 2012;52:1494-505.

19. Rizzo MR, Barbieri M, Marfella R, Paolisso G. Reduction of oxidative stress and inflammation by blunting daily acute glucose fluctuations in patients with type 2 diabetes: role of dipeptidyl peptidase-IV inhibition. Diabetes Care. 2012;35:2076-82.

20. Scheen AJ, Charpentier G, Ostgren CJ, Hellqvist A, Gause-Nilsson I. Efficacy and safety of saxagliptin in combination with metformin compared with sitagliptin in combination with metformin in adult patients with type 2 diabetes mellitus. Diabetes Metab Res Rev. 2010;26:540-9. 